\title{
Transferts de masse et de chaleur entre une gouttelette d'un liquide hygroscopique et l'air humide
}

\author{
R. Pelletret et J.-D. Sylvain* \\ Centre Scientifique et Technique du Bâtiment, Sophia Antipolis, \\ BP 21, 06562 Valbonne Cedex, France \\ (*) CNRS, Laboratoire d'Ecothermique, Sophia Antipolis, BP 21, 06562 Valbonne Cedex, France
}

(Reçu le 25 novembre 1984, révisé les 10 avril et 13 juin 1985, accepté le 18 juin 1985)

\begin{abstract}
Résumé. - Cette étude s'insère dans le cadre des interactions entre un liquide hygroscopique et l'air humide. Par définition, une goutte d'un tel liquide capte les molécules de vapeur d'eau et s'échauffe du fait de la chaleur libérée, à sa surface, par condensation. Des expérimentations, mesurant en régime transitoire la température de gouttelettes de chlorure de lithium et de soude, ont permis de valider, pour nos applications, la corrélation de Ranz et Marshall. Un modèle aux différences finies, de l'évolution en température et en masse d'une goutte d'un liquide hygroscopique dans un flux d'air, est calé sur ces expérimentations. Ce modèle met en évidence le rôle de la diffusivité massique de la phase liquide dans la cinétique de ces transferts et de la taille des gouttes sur l'optimum de température. Il ouvre la voie à une optimisation des échangeurs par pulvérisation d'un liquide hygroscopique dans un flux d'air.
\end{abstract}

\begin{abstract}
This study is a part of a larger research about heat and mass transfer between hygroscopic liquids and moist air. Such a liquid collects water vapour molecules and, because of the heat of condensation, overheats. Transient experiments, measuring the temperature of lithium chloride and soda droplets, led to validate the Ranz and Marshall correlation. A finite difference model of the variation of the temperature and the mass of hygroscopic liquids droplets is compared to those experiments. This model shows off the part of the liquid phase mass diffusivity on the kinetic of the transfers and the part of the droplets dimensions on the maximum temperature. It opens the way to the optimization of exchangers by spraying and hygroscopic liquid in an air flow.
\end{abstract}

\section{Introduction.}

1.1 BUT DE LA RECHERCHE. - La présente étude s'inscrit dans le cadre plus large de la conception optimisée d'échangeurs entre un liquide hygroscopique et l'air.

Les différentes techniques pour réaliser un échange solution hygroscopique-air se classent en trois catégories :

- les échangeurs par ruissellement en film sur des plaques verticales,

- les échangeurs à bulles (formation de bulles d'air au sein du volume liquide),

- les échangeurs par pulvérisation du liquide hygroscopique dans un flux d'air.

L'intensité des échanges entre le liquide et l'air est d'autant plus importante que la surface de séparation des deux milieux est étendue relativement au volume de liquide. Le rapport surface d'échange/volume de liquide (noté $S_{e} / V$ ) dépend du type d'échangeur :

- les films ruisselants présentent un rapport $S_{e} / V$ relativement réduit (de l'ordre de quelques $\mathrm{m}^{2} / \mathrm{m}^{3}$ ) qui n'autorise pas, pour une géométrie classique d'échangeurs, la montée en température du liquide,

- dans les échangeurs à bulles, il est difficile d'obtenir des bulles de très petites tailles et, au cours de leur montée dans le liquide, il se produit une coalescence des bulles qui limite encore plus le ratio $S_{e} / V$, - les échangeurs par pulvérisation se divisent en deux sous-catégories :

- les échangeurs à contre-courant,

- les échangeurs à courants parallèles.

Le dimensionnement des échangeurs à contrecourant est délicat et aboutit à des tailles de gouttes importantes de façon à ce que leur poids compense les forces de viscosité; lorsque les particules sont de tailles importantes (de l'ordre de quelques millimètres de diamètre) le ratio $S_{e} / V$ est équivalent à celui observé dans le cas de ruissellements en films.

Seuls les échangeurs à courants parallèles autorisent, avec une technologie simple, la pulvérisation 
du liquide en fines particules donc l'obtention d'un ratio $S_{e} / V$ important.

La présente étude a pour objet, à terme, la caractérisation des performances des échangeurs par pulvérisation à courants parallèles. Cette caractérisation nécessite la connaissance fine des transferts de masse et de chaleur entre une goutte d'un liquide hygroscopique et l'air humide.

1.2 Présentation de l'étude. - On se propose de modéliser finement les transferts entre une gouttelette et l'air l'environnant. La démarche est la suivante :

- compilation des résultats théoriques et expérimentaux concernant les transferts évapo-condensatifs,

- validation des corrélations, définies à l'étape précédente, à partir de mesures, en régime transitoire, de la température de gouttelettes de liquides hygroscopiques injectées dans l'air humide,

- élaboration d'un logiciel de simulation du comportement, en régime dynamique, de telles gouttelettes,

- mise en évidence de l'influence de certains paramètres thermo-physiques de la phase liquide sur l'intensité des transferts.

\section{Transferts massiques et thermiques entre la surface d'une goutte et l'air.}

2.1 TRANSFerTS DE CHALEUR PAR CONVECTION NATURELLE. - Le transfert de chaleur par convection naturelle entre une goutte et l'air qui l'entoure est dû aux actions conjuguées des différences locales de densité et de l'attraction de la pesanteur. Les actions de la poussee d'Archimède et de la pesanteur s'additionnent ou se contrarient suivant que la goutte est plus chaude ou plus froide que l'ambiance et que l'on considère le transfert convectif sur sa partie supérieure ou inférieure. Le problème est donc au moins bidimensionnel (le nombre de Nusselt dépend de l'angle $\alpha$ (cf. Fig. 1).

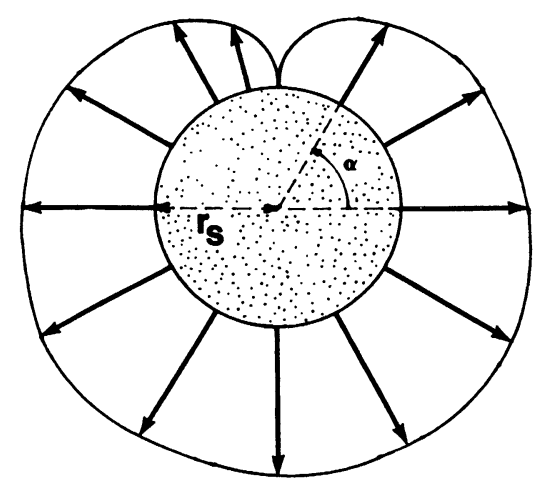

Fig. 1. - Transfert convectif local, d'après la loi de Herman $\left(N u(a)=0,604 G r^{1 / 4} f(a)\right)$, autour d'une sphère se refroidissant dans l'air.

[Heat transfer by natural convection (Herman's law) around a air-cooled sphere.]
Une stylisation des transferts en un modèle monodimensionnel [1] consiste à calculer un nombre de Nusselt moyen pour l'ensemble de la surface sphérique :

$$
N u=\frac{1}{4 \cdot \pi \cdot r_{\mathrm{s}}^{2}} \cdot \iint_{S} N u(\alpha) \mathrm{d} S
$$

$\left\{r_{\mathrm{s}}\right.$ rayon de la goutte

$\{\alpha$ angle mesuré à partir de la position horizontale.

En supposant qu'une description monodimensionnelle des phénomènes suffit au degré de précision recherché, la corrélation semi-empirique suivante [2] s'applique aux cas étudiés :

$$
\mathrm{Nu}=2+0,45 \mathrm{Gr}^{1 / 4} \operatorname{Pr}^{1 / 3}
$$

(valable pour $P r \simeq 1$ et $G r<10^{8}$ )

avec

$$
\left\{\begin{array}{l}
G r=\frac{g \cdot \beta \cdot \Delta T \cdot D^{3}}{v_{\mathrm{a}}^{2}} \\
\operatorname{Pr}=\frac{\eta_{\mathrm{a}} \cdot C_{\mathrm{p}_{\mathrm{a}}}}{\lambda_{\mathrm{a}}}
\end{array}\right.
$$

$g$ attraction de la pesanteur

$\beta$ coefficient de dilatation thermique de l'air

$$
\beta=\frac{1}{\rho_{\mathrm{a}}}\left(\frac{\partial \rho_{\mathrm{a}}}{\partial C}\right)_{T, P}
$$

$\Delta T$ différence de température

$D$ dimension caractéristique

$v_{\mathrm{a}}$ viscosité cinématique de l'air

$\eta_{\mathrm{a}}$ viscosité dynamique de l'air

$C_{\mathrm{p}_{\mathrm{a}}}$ chaleur spécifique à pression constante de l'air

$\lambda_{\mathrm{a}}$ conductivité thermique de l'air.

La dimension caractéristique apparaissant dans les nombres de Nusselt et de Grashof est le diamètre.

La valeur 2 semble être une valeur limite du nombre de Nusselt lorsque le rayon des gouttes tend vers 0 . Cette valeur correspond à une simple conduction à travers une couche d'air au repos, adjacente à la surface de la goutte.

\subsection{TRANSFERTS MASSIQUES PAR DIFFUSION ET CONVEC-} TION NATURELLE. - De la même façon que pour le transfert convectif, le problème du transfert de masse à partir ou vers une sphère peut être stylisé en un problème monodimensionnel par la donnée d'un coefficient moyen d'échanges massiques, $h_{1}$, sur l'ensemble de la surface du liquide.

Différents auteurs ont établi des corrélations empiriques ([3], p. 275-283) liant le nombre de Sherwood aux nombres de Grashof massique et de Schmidt. Citons Garner et Keey (1958) et Steinberger et Treybal (1960). Pour des nombres de Schmidt de l'ordre de celui de l'air $(S c=0,59)$, ces corrélations diffèrent peu et se ramènent à une corrélation antérieure, celle de 
Ranz et Marshall (1952) :

$$
\begin{aligned}
& S h=2+0,6 \mathrm{Grm}^{1 / 4} \mathrm{Sc}^{1 / 3} \\
& \text { (valable pour } \mathrm{Grm}<10^{8} \text { ) }
\end{aligned}
$$

avec :

$$
\left\{\begin{array}{c}
G r m=\frac{g \cdot \beta^{*} \cdot \Delta C \cdot D^{3}}{v_{\mathrm{a}}^{2}} \\
S c=\frac{v_{\mathrm{a}}}{D F_{\mathrm{a}}}
\end{array}\right.
$$

\footnotetext{
( $\beta^{*}$ coefficient de dilatation massique de l'air

$$
\beta^{*}=\frac{1}{\rho_{\mathrm{a}}}\left(\frac{\partial \rho_{\mathrm{a}}}{\partial C}\right)_{T, P}
$$

$\Delta C$ différence de concentration en vapeur d'eau entre l'air au loin et l'air au voisinage immédiat de la surface de la goutte

$D F_{\mathrm{a}}$ diffusivité massique de l'air.
}

La valeur 2 semble, pour beaucoup d'auteurs, être une valeur limite du nombre de Sherwood lorsque le rayon des gouttes tend vers 0 . Une évaluation du coefficient d'évapo-condensation pour de petites gouttes par la voie de la théorie cinétique des gaz montre qu'il n'en est rien.

2.3 INFLUENCE DE LA TAILLE DES GOUTTES SUR LE NOMBRE DE SHERWOOD. - La première loi de Fick conduit à l'expression classique du flux molaire échangé, en régime transitoire, entre une goutte et l'air environnant :

$$
N_{\mathrm{Ar}}=\frac{C_{\mathrm{As}}-C_{\mathrm{A} \infty}}{\frac{r_{\mathrm{s}}}{D F_{\mathrm{a}}}} \text { (loi de Maxwell) }
$$

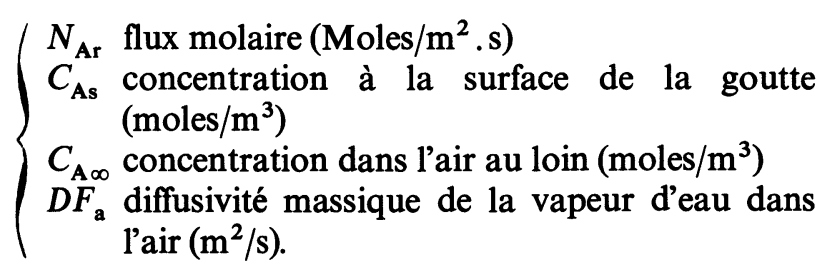

Cette expression permet de retrouver, de façon théorique, la valeur minimale 2 du nombre de Sherwood.

Le flux molaire, calculé à partir de cette expression ne donne des résultats satisfaisants que pour des gouttes suffisamment grosses $\left(r_{\mathrm{s}}>100\right.$ microns).

Lorsque le rayon des gouttes est du même ordre de grandeur que le libre parcours moyen d'une molécule de vapeur d'eau diffusant dans l'air, une description macroscopique des phénomènes n'est plus suffisante. Fuchs [4], en utilisant la théorie cinétique des gaz (théorie de Mayer) a obtenu une expression relativement simp'e du flux de masse diffusant à partir d'une sphère isolée :

$$
N_{\mathrm{Ar}}=\frac{C_{\mathrm{As}}-C_{\mathrm{A} \infty}}{\frac{1}{v . \delta}+\frac{r_{\mathrm{s}}^{2}}{D F_{\mathrm{a}} \cdot\left(r_{\mathrm{s}}+\Delta\right)}}
$$$$
\text { avec : } \quad v=\sqrt{\frac{k \cdot T}{2 \cdot \pi \cdot m}}
$$

( $v$ vitesse statistique moyenne des molécules de vapeur d'eau

$k$ constante de Boltzmann $\left(1,388 \times 10^{-23}\right.$ u.S.I. $)$

$T$ température absolue de l'air

$m$ masse d'une molécule de vapeur d'eau

$$
\left(2,9911 \times 10^{-26} \mathrm{~kg}\right)
$$

$\Delta$ distance depuis la surface de la goutte à laquelle une concentration, pouvant être considérée comme constante, est maintenue

$\delta$ coefficient d'évapo-condensation.

Le coefficient d'évapo-condensation, $\delta$, est défini par:

$$
I=\delta Z S_{\mathrm{g}}
$$

( I nombre de molécules absorbées par la surface de la goutte par unité de temps

$Z$ nombre de chocs à la surface par seconde et par unité d'aire

$S_{\mathbf{g}}$ surface de la goutte.

Cependant, dans l'expression (5), $\delta$ est supposé indépendant du diamètre de goutte et de la température. Frish et Collins [5] ont suggéré qu'une réduction de la taille des gouttes pouvait influer la tension de surface de celles-ci et, par voie de conséquence, la valeur de $\delta$; de plus, les variations de température, dues à la chaleur absorbée ou libérée, peuvent réduire ou accroître la fugacité des molécules de vapeur au voisinage de la goutte et changer ainsi la quantité $\delta$. Okuyama et Zung [6] se sont attachés à déterminer l'influence de la taille des gouttes sur le paramètre $\delta$ et ont obtenu l'expression :

$$
N_{\mathrm{Ar}}=\frac{C_{\mathrm{As}}-C_{\mathrm{A} \infty}}{\frac{1}{v . \delta . \phi\left(r_{\mathrm{s}}\right)}+\frac{r_{\mathrm{s}}^{2}}{D F_{\mathrm{a}} \cdot\left(r_{\mathrm{s}}+\Delta\right)}}
$$

dans laquelle $\phi\left(r_{\mathrm{s}}\right)$ est un coefficient de taille qui s'exprime en fonction du rayon de la goutte par :

$$
\phi\left(r_{\mathrm{s}}\right)=\exp \left(-\frac{3 \cdot v \cdot \sigma}{r_{\mathrm{s}} \cdot k \cdot T}\right)
$$

$\left\{\begin{array}{l}v \text { volume d'une molécule d'eau }\left(2,98 \times 10^{-29} \mathrm{~m}^{3}\right. \\ \left.\text { à } 0^{\circ} \mathrm{C}\right) \\ \sigma \text { tension de surface du liquide }\left(75,2 \times 10^{-3} \mathrm{~N} / \mathrm{m}\right. \\ \left.\text { à } 0^{\circ} \mathrm{C}\right) .\end{array}\right.$ 
Le dénominateur de l'expression (8) comporte deux termes; l'un, $B\left(r_{s}\right)$, est la résistance de la phase gazeuse à la diffusion de la vapeur, l'autre, $C\left(r_{s}\right)$, est la résistance de l'interface liquide-gaz :

$$
\begin{aligned}
& B\left(r_{\mathrm{s}}\right)=\frac{r_{\mathrm{s}}^{2}}{D F_{\mathrm{a}} \cdot\left(r_{\mathrm{s}}+\Delta\right)} \\
& C\left(r_{\mathrm{s}}\right)=\frac{1}{v \cdot \delta \cdot \phi\left(r_{\mathrm{s}}\right)} .
\end{aligned}
$$

La résistance de la phase gazeuse croît lorsque le rayon augmente, alors que la résistance de l'interface varie en sens inverse comme le montre la figure 2 . Il s'ensuit l'existence d'un rayon pour lequel le flux de masse par unité d'aire est maximal (cf. Fig. 3).

Par définition du nombre de Sherwood, il vient :

$$
S h=\frac{h_{1} \cdot\left(2 \cdot r_{\mathrm{s}}\right)}{D F_{\mathrm{a}}} .
$$

Le coefficient de transfert de masse, $h_{1}$, est la somme de l'inverse des résistances à la diffusion de masse :

$$
h_{1}=\frac{1}{B\left(r_{\mathrm{s}}\right)}+\frac{1}{C\left(r_{\mathrm{s}}\right)} \text {. }
$$

Par conséquent, pour de très petites gouttes, c'est-àdire lorsque la migration des molécules de vapeur d'eau au voisinage de la goutte s'effectue par diffusion pure, le nombre de Sherwood prend des valeurs inférieures à 2 (cf. Fig. 4).

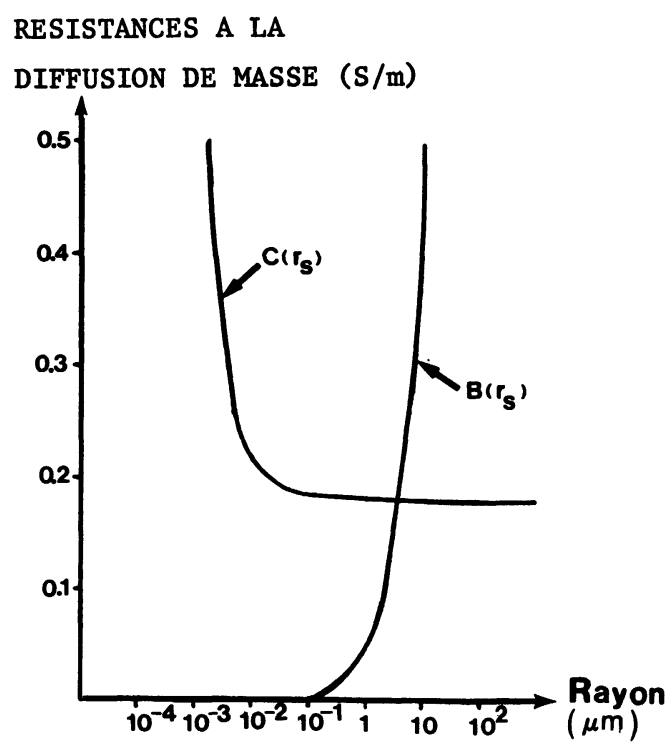

Fig. 2. - Comparaison entre les résistances à la diffusion de masse de la phase gazeuse, $B\left(r_{\mathrm{s}}\right)$, et de l'interface liquidegaz, $C\left(r_{s}\right)$, pour l'évaporation d'une goutte d'eau à $0^{\circ} \mathrm{C}$ dans l'air sec.

[Comparison of diffusional mass resistance of the gasphase, $B\left(r_{\mathrm{s}}\right)$, and of the liquid-gas interface, $C\left(r_{\mathrm{s}}\right)$. Calculations are performed for the evaporation of a water drop in wet air at $0^{\circ} \mathrm{C}$.]

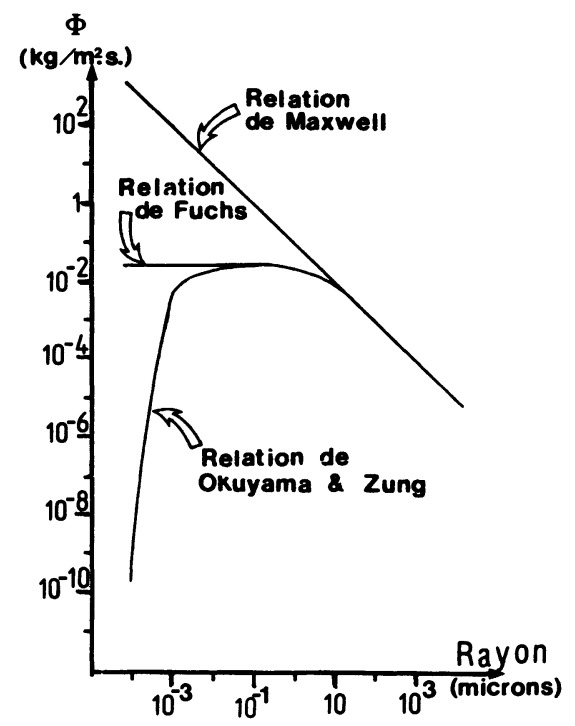

Fig. 3. - Flux massique par unité d'aire et par seconde pour l'évaporation d'une goutte d'eau à $0^{\circ} \mathrm{C}$ dans un air sec.

[Evaporation rate per unit area of water droplets in wet air at $0^{\circ} \mathrm{C}$.]

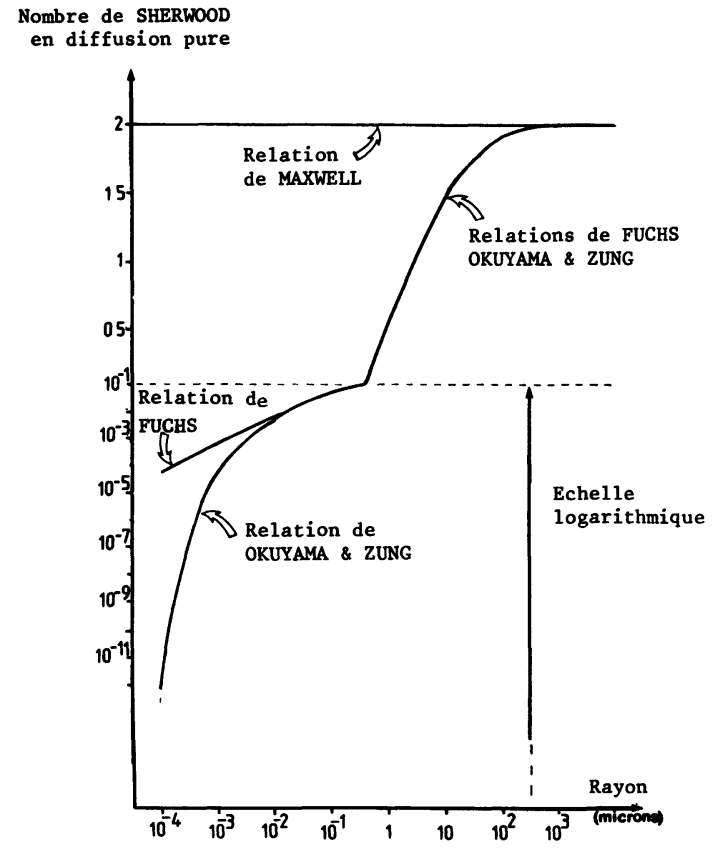

Fig. 4. - Nombres de Sherwood calculés, soit à partir de la théorie classique de la diffusion moléculaire (loi de Maxwell, soit à partir de la théorie cinétique).

[Sherwood numbers calculated either from the Maxwell's law or from the kinetic theory of gases.]

2.4 MOdÈLE DES TRANSFERTS DE MASSE RETENU. En conclusion, nous retenons, pour modéliser les transferts de masse entre la surface d'une goutte et l'air l'environnant, une expression analogue à celle de Ranz et Marshall (3) mais dans laquelle la contribution de la diffusion moléculaire par gradient de con- 
centration, $S h_{\mathrm{d}}$, n'est pas prise égale à 2 mais est calculée à partir de l'expression $(12)$; il vient :

$$
S h=S h_{\mathrm{d}}+0,6 \mathrm{Grm}^{1 / 4} \mathrm{Sc}^{1 / 3} .
$$

\section{Validation de la corrélation de Ranz et Marshall.}

3. 1 BUTS ET DESCRIPTION DES EXPÉRIMENTATIONS. Les expériences consistent à mesurer, en régime transitoire, la température de gouttes de deux liquides hygroscopiques (le chlorure de lithium et la soude).

Elles ont pour buts :

- de valider la corrélation empirique de Ranz et Marshall (obtenue dans des conditions expérimentales particulières) dans le cas de la condensation de vapeur d'eau sur les solutions hygroscopiques étudiées;

- de fournir un ensemble de résultats permettant de caler un logiciel de simulation.

Le dispositif utilisé est décrit figure 5 .

Cet appareillage est placé dans une enceinte climatisée dont la température et l'hygrométrie relative fluctuent, en écarts absolus, de moins de $0,5^{\circ} \mathrm{C}$ et de $2 \%$.

La microburette permet de doser, avec une précision absolue de $0,03 \mathrm{~mm}^{3}$, le volume de solution mis en contact direct avec l'air (cf. Tableau I). Par coulissement du support du thermocouple vers le bas, la goutte est détachée du tube capillaire et se trouve reliée au seul capteur de température. Celui-ci est un thermocoax (Philips) de 250 microns de diamètre. La température de la goutte, celle de l'air ainsi que son degré d'humidité (sonde Vaisala, précision relative $3 \%$ ) sont enregistrés en continu sur un traceur Kipp et Zonen. La précision absolue sur les températures est de $0,1^{\circ} \mathrm{C}$.

Tableau I. - Titres des solutions et gammes des volumes testés.

\section{Composé}

Concentration

(g.Sel/g.Sol.)

Volumes testés $\left(\mathrm{mm}^{3}\right)$

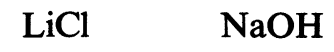

0,41

0,5 à 11
0,485
Les résultats bruts de ces expériences se traduisent par une série de courbes, $T=f(t)$, dont quelques exemples sont donnés figure 6 . Sur ces courbes, on constate une augmentation de la température des gouttelettes, due à la chaleur de condensation, d'autant plus rapide que celles-ci sont petites.

\section{Remarque 1:}

Les gouttes, suspendues au thermocouple, peuvent être assimilées à des systèmes minces au sens des transferts thermiques. En effet, le nombre de Biot relatif à ces transferts est, pour les expériences effectuées, inférieur à 0,1 . Par conséquent, le gradient de

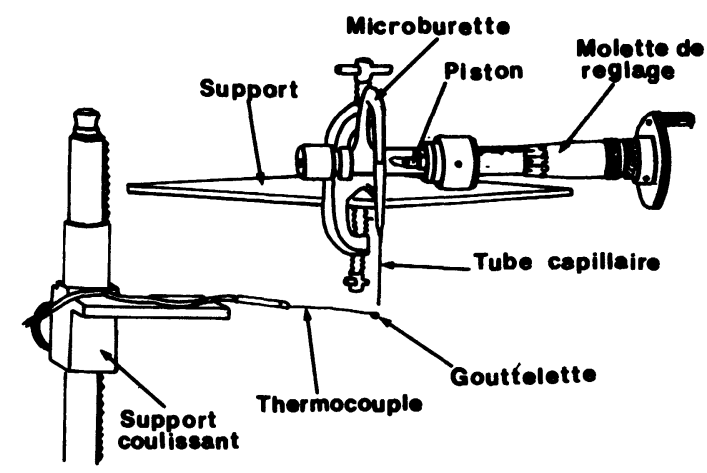

Fig. 5. - Dispositif expérimental destiné à mesurer, en régime transitoire, la température de gouttelettes en convection naturelle.

[Experimental material used to measure, in free convection, the transient temperature of droplets.]

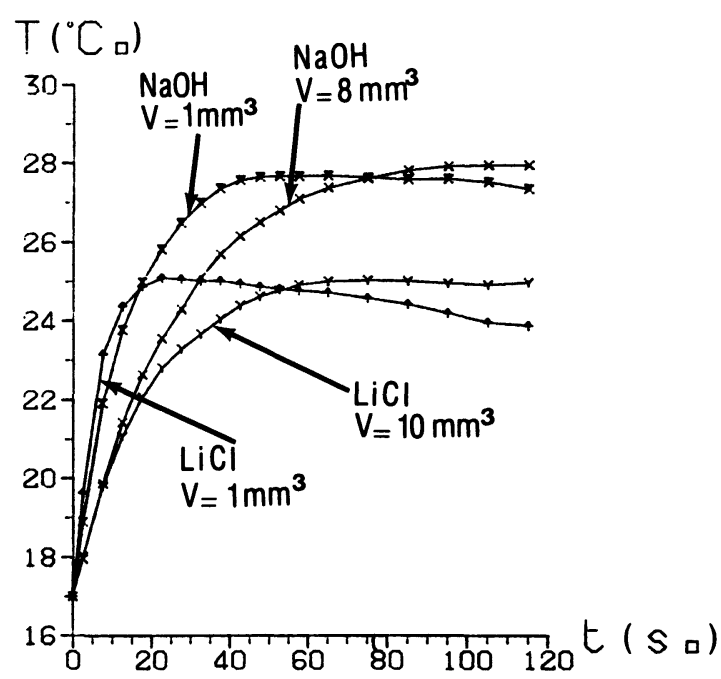

Fig. 6. - Températures mesurées de gouttes de chlorure de lithium et de soude.

[Measured temperatures of some lithium chloride and soda droplets.]

température à l'intérieur du volume de la goutte peut être négligé.

\section{Remarque 2 :}

Le poids de la goutte et les forces de capillarité déforment la sphéricité. Des photographies de gouttes reliées au thermocouple, réalisées au microscope, ont montré que l'ovalisation de la goutte était faible pour des volumes supérieurs à $3 \mathrm{~mm}^{3}$ (accroissement du ratio (surface/volume) inférieur à $10 \%$ par rapport à ce ratio pour une sphère), mais qu'elle pouvait être importante pour de petits volumes (accroissement de $40 \%$ du rapport $\left.S_{e} / V\right)$.

3.2 Calcul du NOMbre de Sherwood. - Le bilan thermique d'une goutte liée au thermocouple, sur un 
intervalle de temps $[t, t+\mathrm{d} t]$, s'écrit :

$$
\begin{aligned}
& \bar{m} \cdot \bar{C}_{\mathrm{p}} \cdot\left[T_{\mathrm{g}}(t+\mathrm{d} t)-T_{\mathrm{g}}(t)\right]= \\
& \quad=E_{\text {ther. }}+E_{\text {rayn. }}+E_{\text {conv. }}+E_{\text {cond. }}+E_{\text {dilu. }}
\end{aligned}
$$

$\bar{m}$ masse moyenne de la goutte sur l'intervalle de temps $[t, t+\mathrm{d} t]$

$\bar{C}_{\mathrm{p}}$ capacité calorifique moyenne du liquide sur l'intervalle de temps $[t, t+\mathrm{d} t]$

$T_{\mathrm{g}}$ température de la goutte

$E_{\text {ther. }}$ énergie échangée par conduction avec le thermocouple

$E_{\text {rayn. }}$ énergie échangée par rayonnement

$E_{\text {conv }}$ énergie échangée par convection

$E_{\text {cond. }}$ énergie libérée par condensation

$E_{\text {dilu. énergie de dilution }}$

Les expressions des différents termes sont :

- en assimilant le thermocouple à une ailette aiguille de grandeur longueur, il vient :

$$
E_{\text {ther. }}=k_{\text {th. }}\left(T_{\mathrm{g}}-T_{\mathrm{a}}\right) \cdot \mathrm{d} t
$$

le coefficient de transfert conductif de ce thermocouple, $k_{\text {th }}$, vaut $0,294 \mathrm{~mW} / \mathrm{K}$;

- en appliquant la loi de Stefan, il vient :

$$
E_{\text {rayn. }}=h_{\mathrm{r}}\left(T_{\mathrm{g}}-T_{\mathrm{p}}\right) \cdot S_{\mathrm{g}} \cdot \mathrm{d} t
$$

avec

$$
h_{\mathrm{r}}=\frac{4 \cdot \sigma \cdot T_{\mathrm{eq}}^{3}}{\frac{1}{\varepsilon_{\mathrm{g}}}+\frac{1}{\varepsilon}-1} \text { et } \quad T_{\mathrm{eq}}=\frac{T_{\mathrm{g}}+T_{\mathrm{p}}}{2} .
$$

\section{Remarque :}

Les gouttes de liquides hygroscopiques sont des milieux semi-transparents; l'émissivité monochromatique directionnelle dépend de l'épaisseur du milieu participant au rayonnement.

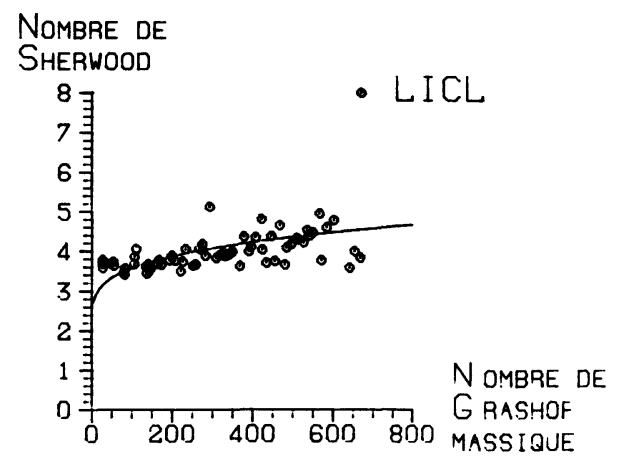

(A)
Devant la non-connaissance des coefficients d'extinction des liquides étudiés, et, considérant le fait que les échanges radiatifs sont faibles comparativement aux échanges convectifs, les pertes radiatives sont calculées par la loi de Stefan en supposant que seule la surface de la goutte rayonne (Emissivité $\varepsilon_{\mathrm{g}}=0,9$ ).

On suppose de plus que les échanges radiatifs s'effectuent avec les parois environnant le dispositif expérimental.

Les températures de ces parois, $T_{\mathrm{p}}$, sont sensiblement égales à la température d'air $T_{\mathrm{a}}$;

- en appliquant la loi de Newton et en supposant valide la relation (2), il vient :

$$
E_{\text {conv. }}=h_{\mathrm{c}}\left(T_{\mathrm{g}}-T_{\mathrm{a}}\right) \cdot S_{\mathrm{g}} \cdot \mathrm{d} t
$$

- la chaleur de condensation est donnée par :

$$
E_{\text {cond. }}=h_{1}\left(C_{\mathrm{g}}-C_{\mathrm{a}}\right) \cdot L_{\mathrm{v}} \cdot S_{\mathrm{g}} \cdot \mathrm{d} t
$$

( $C_{\mathrm{g}}$ concentration massique de la goutte $\left(\mathrm{m}^{3}\right.$ d'eau $/ \mathrm{m}^{3}$ d'air)

$C_{\mathrm{a}}$ concentration massique dans l'air $\left(\mathrm{m}^{3} \mathrm{~d}\right.$ 'eau $/ \mathrm{m}^{3}$ d'air)

$L_{\mathrm{v}}$ chaleur latente de condensation $(2400 \mathrm{~kJ} / \mathrm{l}$ d'eau à $20^{\circ} \mathrm{C}$ )

- la chaleur de dilution est donnée par :

$$
E_{\text {dilu. }}=\mathrm{d} m_{\mathrm{s}} \cdot H_{\mathrm{D}}
$$

$\mathrm{d} m_{\mathrm{s}}$ variation équivalente de la masse en sel de la goutte

$H_{\mathrm{D}}$ énergie de liaison différentielle $(\mathrm{J} / \mathrm{g}$. Sel).

Un programme de calcul pas à pas permet de déduire la valeur de la seule inconnue, $h_{1}$, et donc le nombre de Sherwood calculée par la relation (12).

Les résultats de ces calculs sont portés sur les figures 7A et 7B en fonction du nombre de Grashof massique, le nombre de Schmidt étant égal, dans ces conditions expérimentales, à 0,59 . La comparaison

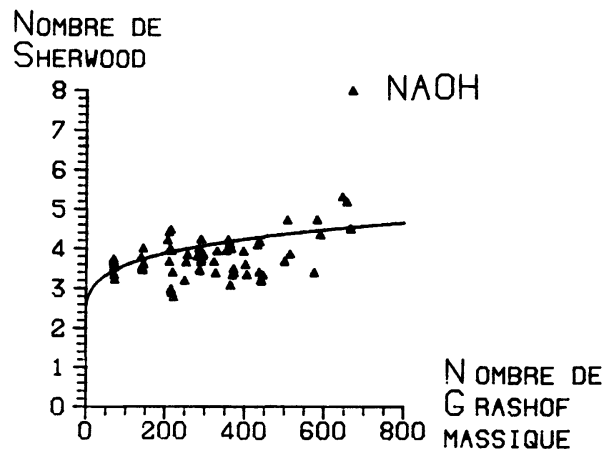

(B)

Fig. 7. - Comparaison entre les nombres de Sherwood calculés à partir des mesures de la température de gouttes isolées et la corrélation de Ranz et Marshall.

[Comparison of Sherwood's numbers calculated from temperatures measurements and the correlation of Ranz and Marshall.] 
des points expérimentaux à la relation de Ranz et Marshall présente, compte tenu des incertitudes liées aux mesures, une bonne corrélation.

\section{Modélisation du comportement en régime dyna- mique de gouttelettes dans un flux d'air.}

On se propose, à terme, de caractériser le comportement d'échangeurs liquide hygroscopique-air à courants parallèles.

Pour ce type d'échangeurs, on suppose que la vitesse d'éjection des particules est suffisamment faible pour que leur vitesse moyenne de déplacement, relativement à un repère fixe, soit égale à la vitesse du flux d'air. La pulvérisation est donc stylisée par un ensemble de $N$ particules, de tailles diverses, influençant chacune un volume d'air proportionnel à leur dimension et au rapport des débits des fluides. Au niveau de chaque particule, les transferts peuvent être décrits de la manière ci-après.

4.1 DESCRIPTION DES PHÉNOMÈNES PHYSIQUES. - Au moment de l'injection dans le flux d'air, la goutte de liquide hygroscopique est supposée homogène. Le gradient de pression partielle de vapeur entre la surface de la goutte et l'air provoque un mouvement diffusionnel des molécules de vapeur d'eau vers cette surface. Ce mouvement peut être accentué ou ralenti par un mouvement d'ensemble de la phase gazeuse dû, par exemple, à un gradient de température.

Leș molécules de vapeur d'eau se condensent à la surface de la goutte; en d'autres termes, elles perdent des degrés de liberté, ce qui se traduit par une libération de chaleur.

Le volume de la goutte, immédiatement adjacent à la surface, s'échauffe et cette chaleur se transmet par conduction-convection vers l'intérieur de la goutte et par convection-rayonnement vers l'ambiance. L'action conjuguée de l'augmentation de la température et de la teneur en eau de la couche surfacique de la goutte tend à accroître la pression partielle à sa surface, tandis que la pression partielle de vapeur de l'air tend à diminuer. $\mathrm{La}$ réduction du différentiel des pressions partielles est d'autant plus rapide que le volume d'air influencé est réduit.

Le phénomène de transfert massique à l'interface se bloquerait instantanément s'il n'y avait migration des molécules d'eau vers l'intérieur de la goutte du fait du déséquilibre de concentration créé en son sein. Plus cette diffusion, de type liquide-liquide, est rapide et plus l'intensité des échanges massiques à l'interface conserve, dans le temps, une valeur importante. La goutte et le volume d'air qu'elle influence évoluent, depuis une situation de non-équilibre thermodynamique, vers une situation d'équilibre dans laquelle les pressions de vapeur à la surface de la goutte et dans l'air se sont égalisées, ainsi que les températures.

4.2 HYPOTHÈSES DE CALCUL. - Le phénomène physique réel est complexe et la description ci-dessus en est déjà une schématisation. Pour pouvoir le décrire mathématiquement, les hypothèses simplificatrices suivantes sont formulées :

1) Les transferts à l'interface sont modélisés par des relations entre nombres adimensionnés.

2) La phase gazeuse est supposée homogène.

3) Les diffusions thermique et massique dans la goutte sont uniquement radiales; le problème est donc à symétrie sphérique.

4) Le processus diffusionnel de l'eau dans la goutte est du type unimolaire unidirectionnel.

5) Les propriétés thermophyșiques des phases ne dépendent que de leurs caractéristiques moyennes.

4.3 VALIDITÉ DES HYPOTHÈSES. - 1) Les corrélations définies au $\S 2$ par les relations (2) et (14) ont été validées expérimentalement.

2) L'utilisation de corrélations entre nombres adimensionnés, pour modéliser les transferts à l'interface, justifie l'hypothèse d'homogénéité de la phase gazeuse. En effet, ces corrélations se réfèrent aux caractéristiques moyennes de l'air. De plus, les mouvements convectifs au sein du volume d'air tendent à homogénéiser, à tout instant, celui-ci.

3) L'hypothèse de diffusion radiale est classique et correspond à une stylisation du problème bidimensionnel en un problème monodimensionnel par calcul d'un coefficient moyen d'échange sur l'ensemble de la surface sphérique.

4) Lorsque le liquide est concentré, il possède une structure quasi cristalline et supposer le transfert massique unimolaire au sein de la goutte est une bonne approximation du phénomène réel.

5) Les valeurs des paramètres thermo-physiques sont recalculées à chaque pas de temps en fonction des valeurs moyennes des températures et des concentrations dans chaque phase. La formulation des calculs s'en trouve simplifiée sans que cela nuise à leur précision.

4.4 Modèle. - En adoptant les notations de la figure 8 et les hypothèses ci-dessus, le système différentiel, décrivant les transferts, en convection naturelle, entre une goutte de liquide et l'air qui l'environne, s'écrit :

- Conditions de symétrie au centre de la goutte $(r=0)$

$$
\left\{\begin{array}{l}
\left(\frac{\partial T_{\mathrm{g}}}{\partial r}\right)_{r=0}=0 \\
\left(\frac{\partial C_{\mathrm{g}}}{\partial r}\right)_{r=0}=0 .
\end{array}\right.
$$

- Diffusion thermique et massique dans le volume de la goutte $\left(0<r<r_{\mathrm{s}}\right)$ :

$$
\left\{\begin{array}{l}
\Delta T_{\mathrm{g}}-\frac{1}{A_{\mathrm{g}}} \cdot \frac{\partial T_{\mathrm{g}}}{\partial t}=0 \\
\Delta C_{\mathrm{g}}-\frac{1}{D F_{\mathrm{g}}} \cdot \frac{\partial C_{\mathrm{g}}}{\partial t}=0 .
\end{array}\right.
$$


- Conditions limites à l'interface $\left(r=r_{\mathrm{s}}\right)$ :

$\left\{\begin{array}{l}\rho_{\mathrm{g}} \cdot C_{\mathrm{p}_{\mathrm{g}}} \cdot \mathrm{d} V_{\mathrm{g}} T \cdot \frac{\partial T_{\mathrm{g}}}{\partial t}=\left(W_{\text {rayn. }}+W_{\text {conv. }}+W_{\text {cond. }}+W_{\text {dilu. }}\right) \cdot S_{\mathrm{g}}-\lambda_{\mathrm{g}} \cdot\left(\frac{\partial T_{\mathrm{g}}}{\partial r}\right)_{r=r_{\mathrm{s}}} \cdot S\left(r-\delta r_{\mathrm{g}} T / 2\right) \\ \mathrm{d} V_{\mathrm{g}} C \cdot \frac{\partial C_{\mathrm{g}}}{\partial t}=J_{\mathrm{s}} \cdot S_{\mathrm{g}}-D F_{\mathrm{g}} \cdot\left(\frac{\partial C_{\mathrm{g}}}{\partial r}\right)_{r=r_{\mathrm{g}}} \cdot S\left(r-\delta r_{\mathrm{g}} C / 2\right)\end{array}\right.$

$W_{\text {rayn. }}$ Flux radiatif échangé entre la goutte et son environnement

$W_{\text {conv. }}$ Flux convectif échangé entre la goutte et l'air

$W_{\text {cond. }}$ Puissance libérée par condensation

$W_{\text {dilu. }}$ Puissance libérée par dilution

$J_{\mathrm{s}} \quad$ Flux moléculaire de vapeur d'eau.

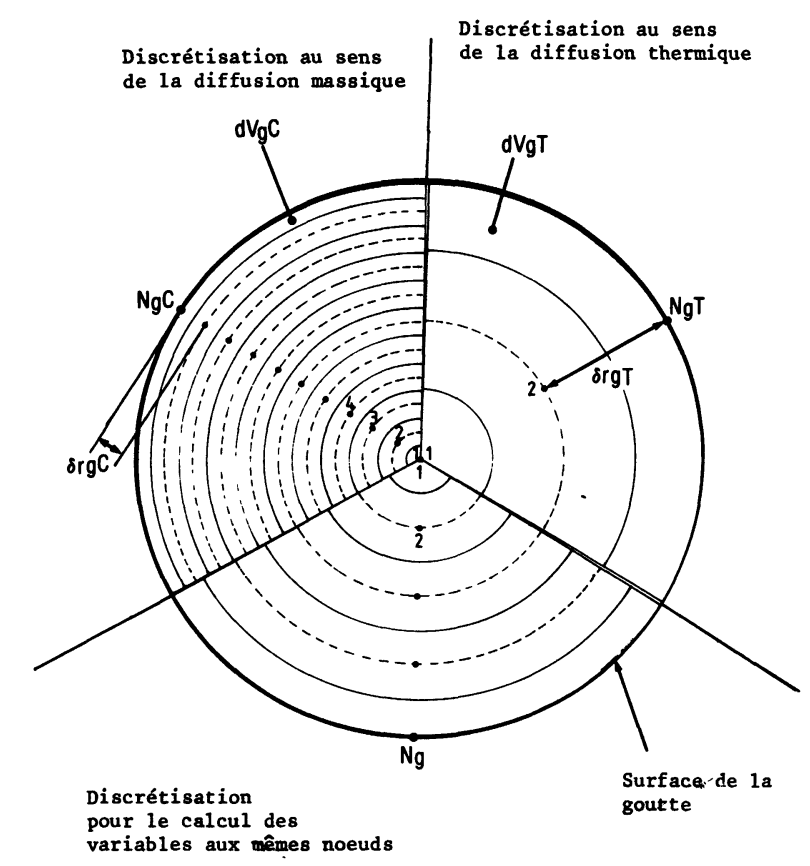

Fig. 8. - Discrétisation spatiale de la goutte au sens des diffusions massique et thermique. Les maillages se superposent. Un troisième maillage permet de calculer températures et concentrations en des lieux identiques.

[Space discretization of a drop, with regard to diffusional mass and heat transfers. The networks are superposed. A third network allows to calculate temperatures and concentrations at the same nodes.]

Les expressions mathématiques des quantités ci-dessus sont identiques à celles explicitées $\S 3.2$ pour les énergies échangées, aux facteurs temps, $\mathrm{d} t$, et surface de la goutte, $S_{\mathrm{g}}$, près.

- Bilans thermique et massique du volume d'air $\left(r_{\mathrm{s}}<\overline{\left.r<r_{\infty}\right):}\right.$

$$
\left\{\begin{array}{l}
\rho_{\mathrm{a}} \cdot C_{\mathrm{pa}_{\mathrm{a}}} \cdot V_{\mathrm{a}} \cdot \frac{\mathrm{d} T_{\mathrm{a}}}{\mathrm{d} t}=\left(-W_{\text {conv. }}\right) \cdot S_{\mathrm{g}} \\
V_{\mathrm{a}} \cdot \frac{\mathrm{d} C_{\mathrm{a}}}{\mathrm{d} t}=\left(-J_{\mathrm{s}}\right) \cdot S_{\mathrm{g}}
\end{array}\right.
$$

- Conditions initiales :

$$
\mathrm{A} t=0\left\{\begin{array}{l}
T_{\mathrm{g}}(r, 0)=T_{\mathrm{g}_{0}} \\
C_{\mathrm{g}}(r, 0)=C_{\mathrm{g}_{0}} \\
T_{\mathrm{a}}(0)=T_{\mathrm{a}} \\
C_{\mathrm{a}}(0)=C_{\mathrm{a}_{0}}
\end{array}\right\} r_{\mathrm{s}}<r<r_{\infty}
$$

4.5 INTÉGRATION DU SYSTÈME D'ÉQUATIONS DIFFÉRENTIELLES. - Des solutions analytiques de ce système existent ([3] p. 21-28, [7] p. 237-238) lorsque des hypothèses supplémentaires peuvent être admises (variation négligeable du volume de la goutte, linéarité des transferts à l'interface, milieux homogènes et isotropes, ...). Dans notre cas, une résolution numérique s'impose (méthode aux différences finies implicite ou explicite). La formulation implicite présente, pour ce système, un inconvénient majeur : les transferts à l'interface étant non linéaires et le lieu des points de discrétisation variant dans le temps, on est contraint de résoudre à chaque pas de temps un système matriciel par itérations successives; cela conduit à des temps de calculs importants. Nous utilisons donc une formulation explicite.

La discrétisation du système différentiel ne présentant aucune difficulté particulière nous n'exposerons pas le détail des calculs (cf. [8] p. 63-68).

Le modèle mathématique constitue le cour du logiciel de simulation qui permet de simuler le comportement de gouttes (sans restriction de taille) de n'importe quelle solution aqueuse, vis-à-vis de la vapeur d'eau contenue dans l'air. Pour cela, il est nécessaire de connaître les caractéristiques thermophysiques du liquide quelle que soit la concentration de celui-ci.

Les résultats de ces simulations sont :

- Les valeurs moyennes des températures et des concentrations dans les deux phases.

- Les gradients dans la phase liquide.

- Les variations de masse de la goutte.

- Les flux échangés à l'interface et l'énergie totale récupérée ou perdue par la goutte. 
4.6 CAlage du logiciel de Simulation. - Le calage du logiciel de simulation consiste à comparer les températures calculées aux températures mesurées, corrigées des pertes dans le thermocouple, de gouttes de liquides testés. Ces comparaisons ont montré que :

- les différences de résultats, entre les simulations supposant la goutte stratifiée en température et celles supposant la goutte isotherme, sont très faibles. En conséquence, dans la plupart des cas, la phase liquide peut être considérée comme un corps mince, au sens de la diffusion thermique;

- la stratification en concentration existe, mais elle ne correspond pas à une diffusion purement moléculaire. En effet, les meilleures corrélations entre résultats expérimentaux et théoriques (cf. Fig. 9) sont obtenues pour des valeurs du module de Fourier supérieures à la limite basse définie par les critères de convergence. Ce qui signifie que la propagation des molécules d'eau au sein du liquide hygroscopique est accélérée par des mouvements internes du volume liquide.

\section{Influence des caractéristiques du liquide sur les trans- ferts entre une gouttelette de ce liquide et l'air}

\section{1 INFLUENCE DE LA DIFFUSIVITÉ MASSIQUE SUR LES} ÉCHANGES. - Le calage du logiciel de simulation a montré que plus la diffusivité massique du liquide est faible et plus la température atteinte par une goutte de ce liquide est basse comparée à la température qu'elle atteindrait si sa diffusivité massique était infinie.

$\mathrm{Au} \S 2.3$, ont été définies les résistances à la diffusion de masse de la phase gazeuse et de l'interface (cf. rela-

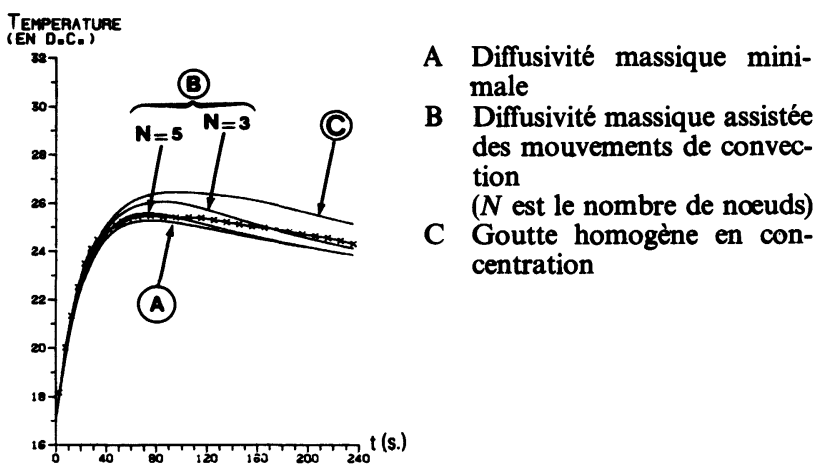

Fig. 9. - Variation en fonction du temps de la température d'une goutte de chlorure de lithium. Comparaison, sur cet exemple, des courbes expérimentales et théoriques $\left(T_{\mathrm{a}}=\right.$ $17^{\circ} \mathrm{C} \mathrm{Hyg}$. $=50 \%$ ).

[Variation of the temperature of a lithium chloride drop with time. Comparison, for that example, of experimental and theoretical curves $\left(T_{\mathrm{a}}=17^{\circ} \mathrm{C}\right.$ Hyg. $\left.=50 \%\right)$.] tions (10) et (11)). En régime dynamique, apparaît une autre " résistance ", celle de la phase liquide. Cette " résistance " caractérise l'aptitude d'un liquide à renouveler sa surface, c'est-à-dire à absorber les molécules d'eau qui s'y condensent, préservant ainsi ses propriétés hygroscopiques. Ceci se traduit par des gradients de concentration d'autant plus accentués que la diffusivité massique est faible : l'examen de la figure 10 montre que la surface d'une goutte de soude se dégrade (au sens de ces propriétés hygroscopiques) beaucoup plus rapidement que la surface d'une goutte de chlorure de lithium (la diffusivité massique de l'eau dans une solution de soude concentrée est trois fois plus faible que la diffusivité de l'eau dans une solution de chlorure de lithium concentrée). Pour caractériser la " résistance " de la phase liquide, nous définissons un coefficient, $\xi$, d'efficacité en température de la solution (dont la concentration est supposée maximale

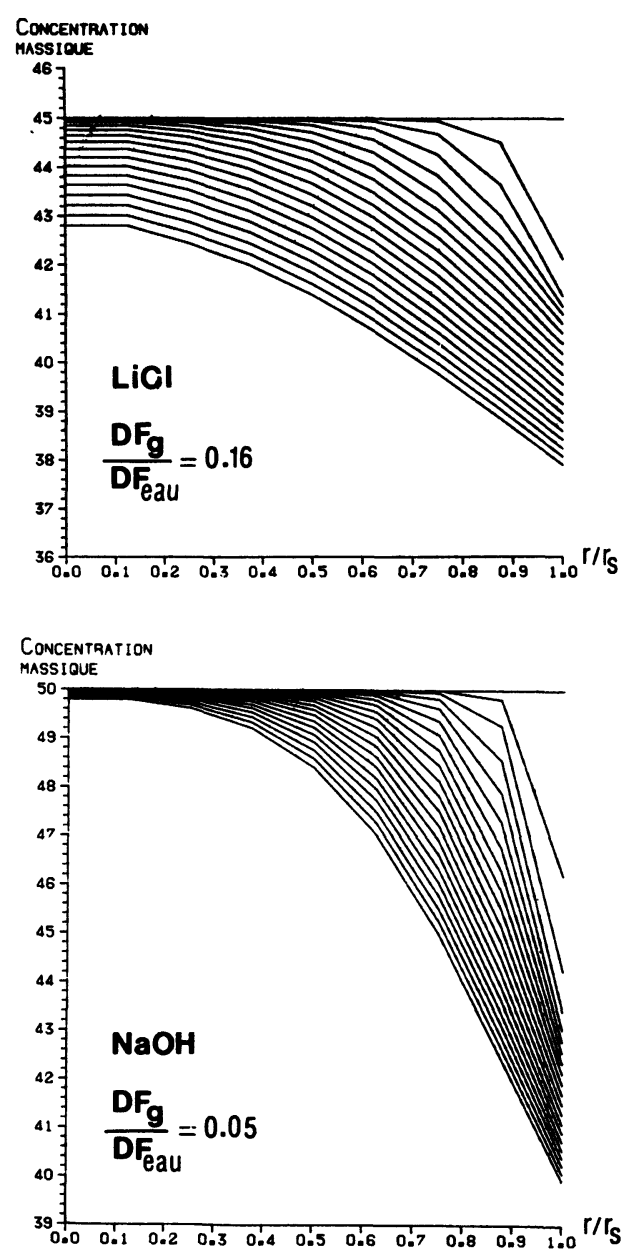

Fig. 10. - Gradients de concentration massique en sel dans des gouttes de chlorure de lithium et de soude $\left(T_{\mathrm{a}}=17^{\circ} \mathrm{C}\right.$ Hyg. $=50 \%$ ).

[Gradients of mass concentration in salt in lithium chloride and soda drops $\left(T_{\mathrm{a}}=17^{\circ} \mathrm{C} \mathrm{Hyg}\right.$. $\left.=50 \%\right)$.] 
à la température ambiante $T_{\mathrm{a}}$ ) par :

$$
\xi=\frac{T_{\max }-T_{\mathrm{g}_{0}}}{T_{\max }^{*}-T_{\mathrm{g}_{0}}}
$$

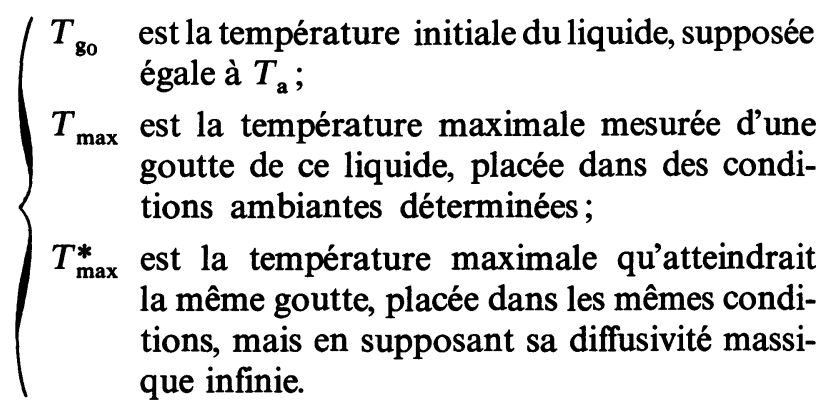

Le coefficient $\xi$ varie peu avec la température ambiante, sa valeur moyenne pour des solutions de chlorure de lithium et de soude est donnée dans le tableau II.

Tableau II. - Coefficient d'efficacité en température des solutions testées.

$\begin{array}{ccc}\text { Composé } & \mathrm{LiCl} & \mathrm{NaOH} \\ \bar{\xi} & - & - \\ & 0,92 & 0,82\end{array}$

\subsection{INFLUENCE DE LA TA.LLE DES GOUTTES SUR L'OPTI-} MUM DE TEMPÉRATURE. - Expérimentalement, nous avons constaté une légère influence de la taille des gouttes sur la température maximale atteinte, mais l'intervalle de variation des volumes était réduit $(1$ à $10 \mathrm{~mm}^{3}$ ). Le logiciel de simulation permet d'étendre l'étude de l'influence de la taille des gouttes à un large intervalle de volumes (cf. Fig. 11). Il ressort que :

- lorsque la taille des gouttes excède $1 \mathrm{~mm}^{3}$, leur température maximale ne dépend plus que très peu du volume (moins de $2 \%$ d'écart entre les températures atteintes par une goutte de $1 \mathrm{~mm}^{3}$ et par une goutte de $20 \mathrm{~mm}^{3}$ );

- en deçà de cette valeur, la température maximale décroît rapidement avec le volume. Ce phénomène est lié à l'accroissement de la résistance à la diffusion de masse de l'interface.

\section{Conclusion.}

- Cette étude a montré que la diffusivité massique de la phase liquide joue un rôle dans l'intensité des transferts de masse en régime transitoire. La notion classique d'hygroscopicité peut être complété par une notion nouvelle de " pouvoir hygroscopique " prenant en compte la cinétique des transferts couplés de masse et de chaleur.

Un liquide, réputé fortement hygroscopique du fait que sa pression partielle, mesurée à l'équilibre,

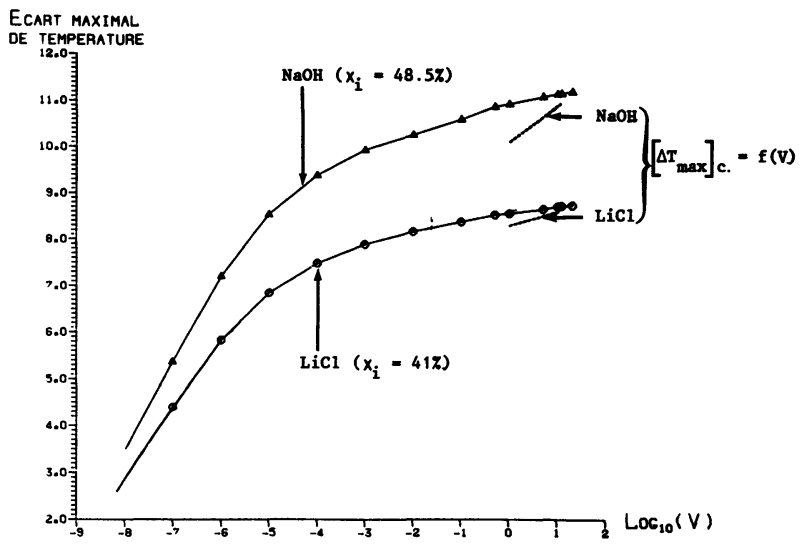

Fig. 11. - Influence de la taille des gouttelettes sur leur température maximale (conditions ambiantes $T_{\mathrm{a}}=17^{\circ} \mathrm{C}$ Hyg. $=50 \%$ ). Les droites $\Delta T_{\max }=f(V)$, obtenues par régression linéaire à partir des expérimentations, présentent des pentes plus importantes que les pentes moyennes théoriques sur l'intervalle $\left[1 \mathrm{~mm}^{3}, 10 \mathrm{~mm}^{3}\right]$; ceci est dû aux variations de certains paramètres (température et hygrométrie de l'air, concentration initiale des solutions) au cours des expériences.

[Plot of the maxima temperatures reached by soda or lithium chloride droplets versus their radius. The slopes of the experimental lines, calculated by linear regression, are different of the average theoretical slopes on the interval $\left[1 \mathrm{~mm}^{3}, 10 \mathrm{~mm}^{3}\right]$, because of the variations of experimental parameters such temperature and hygrometry of air and initial concentration of solutions.]

est faible, peut s'avérer moins performant (au sens de la masse de vapeur d'eau captée sur un intervalle de temps donné) qu'un autre liquide dont la pression partielle, à l'équilibre, est plus élevée mais dont la diffusivité massique est plus importante.

Ce dernier liquide stocke en son sein les molécules de vapeur d'eau collectées à sa surface. Les molécules de sel, en surface, restent peu hydratées. Ce liquide conserve ainsi, dans le temps, un pouvoir hygroscopique important.

- La modélisation précise des échanges de masse entre une gouttelette d'un liquide hygroscopique et l'air doit permettre une optimisation des échangeurs de déshumidification, au sens du meilleur compromis entre le choix du liquide, les débits des fluides et la granulométrie (donc le choix des buses).

- La mise en évidence de l'accroissement important de la température du liquide lors de l'échange de masse avec un flux d'air ouvre la voie à des applications de production de chaleur, par exemple par couplage entre un échangeur atmosphérique par pulvérisation d'une solution hygroscopique et une pompe à chaleur. 


\section{Bibliographie}

[1] HeRman, R., Warmeübergang bei freier Ströhmung am wagrechten Zylinder in zwei-atomic Gasen. Forshungsheft (1936) N. 379.

[2] HuETZ, M., Convection naturelle (Cours E.C.P.) 1975.

[3] Skelland, A. H. P., Diffusional mass transfer (Wiley Interscience Publication) 1974.

[4] Fuchs, N. A., Physik Z. Sowjetunion 6 (1934) 224.

[5] Frish, H. L. et Collins, F. C., J. Chem. Phys. 21 (1953) 2158.
[6] Oxuyama M. et Zung, J. T., J. Chem. Phys. 46 (1967) 1580.

[7] Carslaw, H. S. et Jaeger, J. C., Conduction of heat in solids (2nd edition).

[8] Pelletret, R.-Y., Contribution à l'étude des transferts couplés de masse et de chaleur entre une gouttelette d'un liquide hygroscopique et l'air. Conception d'échangeurs par pulvérisation d'un liquide dans un flux d'air. Thèse de Docteur-Ingénieur, Université de Valenciennes (1984). 